\title{
Evaluation of the clinical utility, efficacy and safety of a novel medical device for the treatment of foot ulcers: Rationale and design of the carbothera trial
}

\author{
Paramjit S. Tappia $\mathrm{PhD}^{1}$, Grant N. Pierce, $\mathrm{PhD}^{2}$, Bram Ramjiawan, $\mathrm{PhD}^{3}$, Hiroaki Hasebe DVM MVSc${ }^{4}$, Hideo Kumamoto DVM ${ }^{5}$
}

\begin{abstract}
Tappia PS, Pierce GN, Ramjiawan B, Hasebe H, Kumamoto H. Evaluation of the clinical utility, efficacy and safety of a novel medical device for the treatment of foot ulcers: Rationale and design of the carbothera trial. Curr Res Cardiol 2016;3(4):117-120.

BACKGROUND: Foot ulceration usually precedes more serious foot complications such as infection, gangrene or amputation. The risk of developing foot ulcers has been estimated to be higher in individuals with peripheral arterial disease, diabetes and kidney disease.

OBJECTIVE: To evaluate the healing potential of $\mathrm{CO}_{2}$-enriched water in patients with foot ulceration.

METHODS: This is a multi-centered; placebo controlled and randomized clinical trial. Patients will have at least 1 distal extremity ulcer and will be randomized to receive a treatment with bath therapy at $37^{\circ} \mathrm{C}$ containing either 1,000-1200 ppm $\mathrm{CO}_{2}$-enriched tap water (the intervention), or noncarbonated tap water (the control group). The treatment will be carried out 3 times/week for 15 minutes per session for 4 months for a total of 48 treatments. At baseline and at the end of every treatment month, wound
\end{abstract}

assessment, measurement of limb oxygenation, ankle brachial pressures as well as McGill pain questionnaire will be carried out. Blood will also be collected at baseline and at the end of every month of the treatment period (for a total of 5 collections) for the measurement of different biomarkers. BASELINE DATA: At baseline all 14 participants have at least one distal extremity ulcer and $93 \%(13 / 14)$ are diabetic. Although 6 of the participan 'ts have peripheral arterial disease (left and/or right limb) as evidenced by an ankle brachial index (ABI) $<0.9$, another 5 exhibited non-compressible ankle arteries and 3 participants with left and right $A B I$ of $\geq 0.9$. Mean age of participants is $60.2 \pm 1.7$ and $64 \%(9 / 14)$ of the participants are undergoing hemodialysis for ESRD and 29\% (4/14) are below knee amputees.

CONCLUSIONS: The proposed clinical study will evaluatve the therapeutic potential, safety and efficacy of $\mathrm{CO}_{2}$-enriched water (produced by the Carbothera device) on treating foot ulcers.

Key Words: Foot ulcers; Diabetes; Peripheral arterial disease; Hemodialysis; Foot bathing, $\mathrm{CO}_{2}$-enriched water
$\mathrm{T}$ he risk of developing foot ulceration has been estimated to be $25 \%$ higher in individuals with diabetes (1), representing a huge economic burden on health care costs (2). Bacterial infection, tissue ischemia, continuing trauma, and poor management cause diabetic foot ulcers to heal slowly and transform into chronic wounds (3). Critical limb ischemia (CLI) is a severe form of peripheral arterial disease (PAD). PAD, commonly affecting tibial and peroneal arteries of the calf, contributes to the development of foot ulcers in up to $50 \%$ of cases. Among other factors, the vasoconstriction secondary to a decrease in endothelium-derived vasodilators plays a central role in the pathophysiology of foot ulcers (4). The aims of management in patients with CLI are to relieve ischemic pain, heal ulcers, prevent limb loss, improve function and quality of life and prolong survival (5). Dialysis is a risk factor for foot complications in patients with diabetes and chronic kidney disease (CKD) (6). Indeed, patients with diabetes and CKD receiving dialysis have a 5 -fold higher prevalence of foot ulceration as compared to pre dialysis diabetic patients (7). It has also been observed that the prevalence of other lower limb complications (amputation, PAD, prior ulcer and neuropathy) was approximately 2 -fold higher in patients receiving dialysis. Foot ulceration usually precedes more serious foot complications such as severe infection, gangrene or amputation. In a retrospective study involving 90 patients with diabetes who started dialysis, it was found that the cumulative incidence of foot ulceration and amputation increased before the initiation of dialysis and then was highest in the subsequent 2 years (8).

During hemodialysis there is a reduction in skin microcirculation and tissue oxygenation that may contribute to the development of ulceration in patients with diabetes (9). In this regard, some studies have shown an improvement in peripheral blood flow in patients with different stages of
PAD in response to Carbothera treatment (10-15). It is known that $\mathrm{CO}_{2}$ causes vasodilation and thus immersing the extremities into carbonated water will increase skin blood flow, given that $\mathrm{CO}_{2}$ can permeate through the skin (15). The Carbothera device has also been examined in diabetic foot ulcers $(16,17)$. However, in view of the limitations of case studies, there is a need for a well-designed clinical trial to determine the efficacy of $\mathrm{CO}_{2}$-enriched water $(1000-1200 \mathrm{ppm})$ produced by the Carbothera device to relieve foot ulcers. Moreover, despite the fact that multiple treatments have been studied and used for ulcer healing (18), only $24-50 \%$ of all ulcers will heal after 12 weeks to 6 months of standard treatment $(19,20)$. The proposed clinical trial will, therefore, evaluate the therapeutic potential of $\mathrm{CO}_{\text {-enriched water in }}$ patients with foot ulceration. Accordingly, this study will test the hypothesis that $\mathrm{CO}_{2}$-enriched water bath treatment generated by the Carbothera device will reduce foot ulcer size. The general objective of the study is to evaluate determine peripheral blood flow in patients to evaluate the efficacy of $\mathrm{CO}_{2}$ -enriched water on treating foot ulcers in patients. The specific objectives of the study are to:

- Determine peripheral blood flow in patients following $\mathrm{CO}_{2}$-enriched water treatment.

- Determine ulcer size and rate in response to $\mathrm{CO}_{2}$-enriched water treatment.

- Investigate limb oxygenation in response to $\mathrm{CO}_{2}$-enriched water treatment.

- Determine the effect of $\mathrm{CO}_{2}$-enriched water treatment on biomarkers of angiogenesis, inflammation, as well as lipid profile, fasting blood glucose and HbA1c.

${ }^{1}$ St Boniface Hospital, Asper Clinical Research Institute and ${ }^{2}$ Department of Physiology and Pathophysiology, Max Rady College of Medicine, Rady Faculty of Health Sciences, University of Manitoba, Winnipeg, Canada, ${ }^{3}$ Department of Pharmacology and Therapeutics, Max Rady College of Medicine, Rady Faculty of Health Sciences, University of Manitoba, Winnipeg, Canada, ${ }^{4}$ Mitsubishi Rayon America Inc., New York, USA, ${ }^{5}$ Mitsubishi Chemical Holdings, Tokyo, Japan

Correspondence: Dr. Paramjit S. Tappia, Asper Clinical Research Institute, CR3129-369 Tache Avenue, Winnipeg, Manitoba, Canada R2H 2A6, Telephone 204-2581230, e-mail ptappia@sbrc.ca

Received: August 12, 2016, Accepted: August 16, 2016, Published: August 18, 2016 


\section{METHODS}

Study Design

This is a multi-centered; placebo controlled and randomized clinical trial. Study participants will be patients with foot ulceration. The inclusion and exclusion criteria are shown in Table 1.

\section{TABLE 1}

\section{Inclusion and exclusion criteria}

\section{Inclusion:}

1. Male and female participants $\geq 18$ years of age with at least one distal extremity ulcer (arterial ischemic ulcer).

2. No acute medical illness and on conventional medical management prior to Carbothera.

3. Willingness and ability to provide written informed consent

\section{Exclusion:}

\section{DVT or PE within the past 12 months.}

2. Subjects with known active cancer, HIV, hepatitis $B$ virus, hepatitis $C$ virus, human transmissible spongiform encephalopathy, Treponema pallidum.

3. Subjects who are deemed to have an infection of the distal extremity ulcer.

4. Pregnant women.

5. Unstable angina.

6. Acute MI within 1 month.

7. Stroke within 1 month.

8. Patient scheduled for revascularization during the 4-month intervention period.

Patients will be randomized to receive a foot bath treatment with either $\mathrm{CO}_{2}$-enriched tap water (1,000-1200 $\mathrm{ppm} \mathrm{CO}_{2}$ (the treatment group), or non $\mathrm{CO}_{2}$-enriched tap water (the control group). The Carbothera ${ }^{\mathrm{TM}}$ is a device used to generate $\mathrm{CO}_{2}$-enriched water (Figure 1).

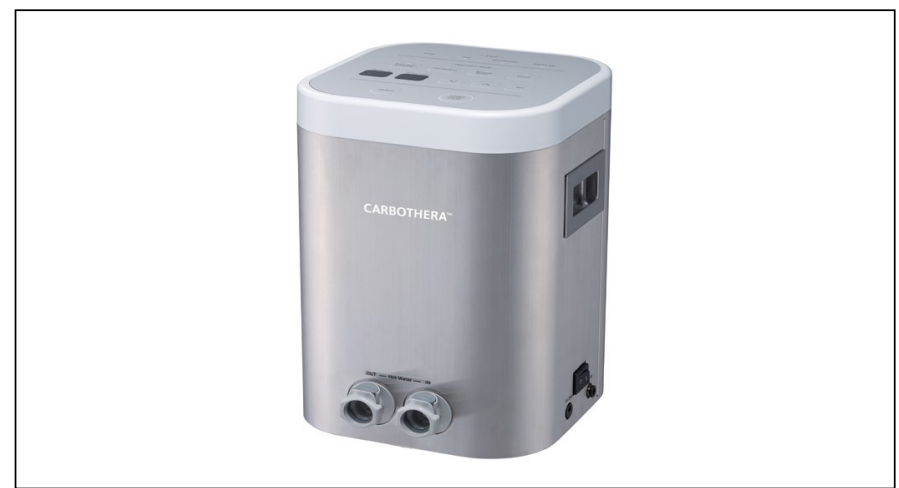

Figure 1) Carbothera device for foot bathing

The use of this device does not involve contact with the patient. A major component of the device is the gas-permeable hollow fibre $\left(\mathrm{GPH}^{\mathrm{TN}}\right)$ membrane developed by Mitsubishi Rayon Cleansui, Tokyo, Japan. The membrane is composed of a non-porous ultra-thin film with gas-selective permeability that is sandwiched between two porous layers. The $\mathrm{GPH}^{\mathrm{TM}}$ membrane conveniently produces large quantities of $\mathrm{CO}_{2}$-enriched water (Figure 2).

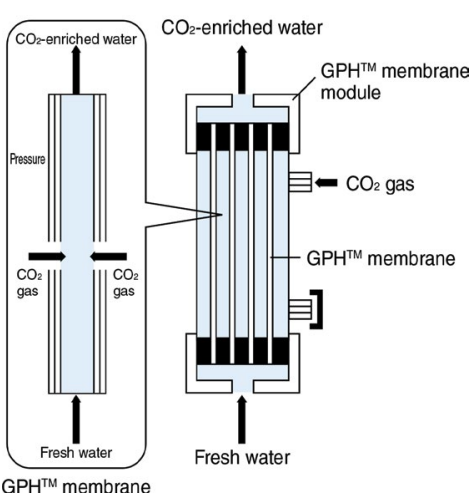

B:

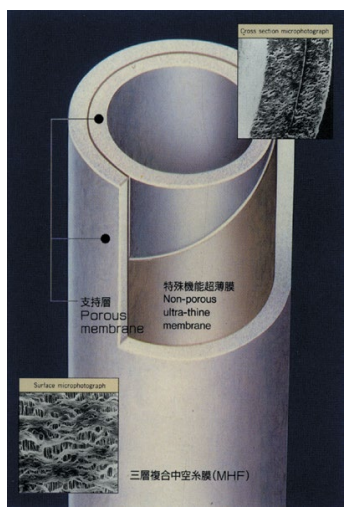

Figure 2) The unique Carbothera membrane system for producing $\mathrm{CO}_{2}$ in a rapid and inexpensive manner. Panel A shows the structure of the GPHTM membrane developed by Mitsubishi Rayon Cleansui; Panel B shows $\mathrm{CO}_{2}$. enriched water production with the GPHTM membrane

The middle layer of the $\mathrm{GPH}^{\mathrm{TM}}$ membrane allows $\mathrm{CO}_{2}$ to pass through, but is impermeable to water. $\mathrm{CO}_{2}$ on the high-pressure side diffuses through the molecular structure of the thin film and dissolves into the water on the low-pressure side. A large number of $\mathrm{GPH}^{\mathrm{TM}}$ membranes can be bundled together into a module, which can be used to produce a large amount of water containing a high concentration of dissolved $\mathrm{CO}_{2}$. The bath therapy will be carried out at $37^{\circ} \mathrm{C}, 3$ times/week, for 15 minute sessions for a total of 4 months. Blood (approximately $20 \mathrm{ml}$ ) will also be collected at baseline and at the end of every month of the treatment period (for a total of 5 collections) for the measurement of the different biomarkers.

\section{Participant Selection, Recruitment and Informed Consent}

Participants will be screened for eligibility and then approached for written informed consent before enrolment into the study. Patient demographics (diabetes, PAD, hemodialysis) will be recorded at screening. Participants will be given both written and verbal information describing the nature of the study. Sufficient time will be allowed for participants to provide their consent. The procedures for conducting informed consent will be documented in the study records and a copy of the signed consent will be given to the participants enrolled in the study. Information on participant eligibility will also be obtained from the individual's medical history.

\section{Foot Bathing}

Participants will undergo foot bathing with $\mathrm{CO}_{2}$-enriched tap water $\left(37^{\circ} \mathrm{C}\right.$; $\mathrm{CO}_{2}$ concentration of $1000-1200 \mathrm{ppm}$ ) or a non- $\mathrm{CO}_{2}$-enriched tap water $\left(37^{\circ} \mathrm{C}\right)$ with an immersion time of 15 minutes, 3 times per week for 4 months for a total of 48 treatments. Participants will be rested on a chair for $15 \mathrm{~min}$ before foot bathing. The primary and secondary outcome measures are shown in Table 2 .

\section{TABLE 2}

\section{Primary and secondary outcome measurements}

\begin{tabular}{l}
\hline Primary outcome measures: \\
\hline 1. Ulcer area (length $x$ width). \\
2. Ulcer volume (length $x$ width $x$ depth). \\
3. Formation of new ulcers. \\
4. Ulceration rate. \\
5. Ankle-brachial index/Blood flow by Doppler. \\
\hline Secondary outcome measures: \\
1. Degree of rest pain. \\
2. Limb oxygenation by a non-invasive near infra-red spectroscopy. \\
3. Angiogenesis marker: vascular endothelial growth factor (VEGF). \\
4. Inflammatory markers: TNFa, IL-6 and C-reactive protein. \\
5. Markers of glucose handling: HbA1c and fasting blood glucose. \\
6. Lipid profile: Total cholesterol, HDL-cholesterol, LDL-cholesterol, triglycerides. \\
7. Use of bespoke footwear. \\
8. Ability to walk barefoot at home. \\
9. Incidence of amputation. \\
10. Use of antibiotics indicated for the ulcer treatment.
\end{tabular}

In order to achieve these, the following procedures will be conducted 5 times: at baseline, 1, 2, 3 and 4 months.

Measurement of ulcer size

The Silhouette Mobile camera (ARANZ Medical, Christchurch, New Zealand) will be used to measure ulcer area, volume and depth. This device is a portable handheld computer with an integrated high-resolution digital camera and an embedded laser light. The device has been shown to be accurate to within $2 \%$ of surface area on wound models and has demonstrated intraand inter-user variability of only $2-5 \%$ in clinical use (21).

\section{Ankle/Brachial Index (ABI)/Blood flow}

A continuous wave, hand held Doppler machine will be used with a blood pressure cuff and a conventional sphygmomanometer to measure the systolic pressure in both the posterior tibial (PT) and dorsalis pedis (DP) arteries. If no PT or DP arterial signals are found, the anterior tibial and/or peroneal arterial pressure will be recorded. The ankle systolic pressure and brachial systolic blood pressure will be calculated as ankle systolic pressure/brachial systolic pressure (ABI). The subject will be asked to rest supine for 10 minutes before ABI measurements. Brachial systolic and diastolic pressures as well as heart rate will also be measured. The ABI has a high level of sensitivity 
and specificity for diagnosing PAD (22). In the event that PT/DP are noncompressible, toe pressures by photoplethysmographic (PPG) technique will be measured.

\section{Limb oxygenation}

Changes in limb blood volume and oxygenation will be recorded using noninvasive near infra-red spectroscopy (NIRS) (23-26). NIRS measures the percentage of hemoglobin oxygen saturation in the microcirculation of tissue up to $3 \mathrm{~cm}$ below the skin (24)

\section{Biochemical measurements}

Blood samples from participants will be analyzed for C-reactive protein, glucose, total cholesterol, triglycerides, high density lipoprotein (HDL)cholesterol and low density lipoprotein (LDL)-cholesterol levels using standard methods by Laboratory Services at St. Boniface Hospital operated by Diagnostic Services of Manitoba. TNF- $\alpha$, Il- 6 and VEGF will be measured by ELISA method in the Albrechtsen Research Centre at St Boniface Hospital in Winnipeg, Canada as described elsewhere (27).

\section{Questionnaires}

The short form McGill Pain Questionnaire (28) will be used to assess the degree of rest pain. A questionnaire will also be used to determine the use of other therapies. A patient self-report for the following will be conducted: use of bespoke footwear or insoles and ability to walk barefoot at home. Use of antibiotics indicated for the ulcer treatment during the period of the trial will also be recorded in the questionnaire. Responses to these questions will be recorded as Yes (with frequency noted) or No at baseline and at the end of 2 and 4 months of treatment (7).

\section{Safety/Rescue Medication}

Since this is not a drug study, rescue medication is not warranted. No adverse effects are expected based upon literature and prior experience. Should any participant experience adverse effects as a consequence of their participation in the study, they will be excluded from the study and receive routine medical care. However, any adverse events, as identified by the WHO scale, will be followed up. Research staff will record final outcome and resolution date of the event wherever possible. All serious adverse events (representing a significant health hazard to the participant) will be reviewed by the monitor and reported in an expedited manner to Health Canada, the Research Review Committee of St. Boniface Hospital and the University of Manitoba Research Ethics Board.

\section{Premature Withdrawal/Discontinuation Criteria}

Participants are entitled to withdraw from study at any time for any reason. In addition, the study will be halted at any time should the data safety and monitoring committee deem it harmful to the participants to continue with the trial. The clinical investigator may withdraw any participant from the study at any time for medical reasons, or if the participant is consistently and continually non-compliant with the study protocol.

\section{Statistical Analysis}

Previous analyses exploring the effectiveness of $\mathrm{CO}_{2}$-enriched water as compared to non-carbonated water for improving limb function following continuous treatment have shown large positive effects in favor of $\mathrm{CO}_{2}$ enriched water for both changes in microcirculation; difference $=2.20$ standard deviations (12) and ulcer size (16); Difference $=0.70$ standard deviations). A final sample size of 40 individuals will allow for the detection of a standard deviation difference of 0.90 for in ulcer area/volume, blood flow, and ankle-brachial index as measured by Doppler at $80 \%$ power and $5 \%$ alpha. This sample size will also allow for large absolute differences (35\%) to be detected in the formation of new ulcers, and the ulceration rate at $80 \%$ power and 5\% alpha.

A repeated measures analysis of variance (ANOVA) will be utilized to discriminate differences in the continuous outcome variables within the experimental group across the sampling time period (baseline, 1, 2, 3 and 4 months). Where significant within-subject differences are found, a NeumanKuels post-hoc test will be used to compare specific mean scores across the repeated measures time factor. Event rate differences for all dichotomous variables will be analyzed using Kaplan Meier Survival Curves with a followup period of four months. For all statistical analyses, the significance level will be set at $\mathrm{P}<0.05$.

\section{Confidentiality of Data}

All information collected for the purpose of the study will be kept in a locked and secured area. All information collected and sent for statistical analysis will only have the participant's initials and study number. Only the research personnel will be able to link the study identifiers and the participant's name. All identifiers will be treated in confidence and in accordance with the Personal Health Information Act of Manitoba. Study records will be retained for 25 years. For the purpose of trial-related monitoring/auditing, the University of Manitoba Research Ethics Board and the St. Boniface Hospital Research Review Committee will have access to source data and study records.

\section{Ethics Approval}

Written approvals from the Research Ethics Board at the University of Manitoba and St. Boniface Hospital Research Review Committee have been obtained prior to implementation of the study and enrollment of any participants. The tenets of Good Clinical Practice will also be followed in the conduct of this study.

\section{Essential Study Documents and Study Access}

The Principal Investigator accepts responsibility for the overall conduct of the study and for the documents that are essential for the conduct of the study. Any documents which reveal the identity of the patient (such as the consent form) will be stored in the Principal Investigator's office at St. Boniface Hospital in a locked cabinet. Direct access to all documents associated with this study will be permitted in the case of auditing by the University of Manitoba Research Ethics Board and St. Boniface Hospital. Archiving will be done as per the standard regulatory and institutional requirements.

\section{RESULTS}

\section{Baseline data}

Some of the baseline characteristics of the 14 participants that have, to date, been compliant with the baseline visit, are presented in Table 3 .

\section{TABLE 3}

Baseline characteristics of patients enrolled into the Carbothera Study

\begin{tabular}{ll}
\hline Participants (n) & $\mathbf{1 4}$ \\
\hline Age (years) & $60.2 \pm 1.7$ \\
Sex (\% M/F) & $64 \mathrm{M}(9) ; 36 \mathrm{~F} \mathrm{(5)}$ \\
Diabetes (\%) & $93(13)$ \\
Hemodialysis (\%) & $64(9)$ \\
Fasting Blood Glucose (mM) & $10.30 \pm 1.54$ \\
HbA1c (\%) & $8.81 \pm 0.55$ \\
Cholesterol (mM) & $4.02 \pm 0.27$ \\
Triglycerides (mM) & $1.90 \pm 0.46$ \\
HDL-cholesterol (mM) & $1.04 \pm 0.12$ \\
LDL-cholesterol (mM) & $2.10 \pm 0.22$ \\
Total cholesterol/HDL-cholesterol ratio & $3.95 \pm 0.36$ \\
LDL-cholesterol/HDL-cholesterol ratio & $2.21 \pm 0.31$ \\
C-reactive Protein (mg/L) & $15.05 \pm 4.25$ \\
\hline
\end{tabular}

Values are means \pm S.E. of 13-14 independent measurements. Reference range for fasting glucose, 3.6-6.0 mM; HbA1c, 4.0-6.0\%; cholesterol, < $5.2 \mathrm{mM}$; triglycerides, < $1.7 \mathrm{mM}$; HDL-cholesterol, > 1.0 $\mathrm{mM}$; LDL-cholesterol $<3.4 \mathrm{mM}$; total cholesterol/HDL-cholesterol $<4.5 \mathrm{mM}$; LDL-cholesterol, <3.5 mM; C-reactive protein, $<5.0 \mathrm{mg} / \mathrm{L}$.

The population has an average age of 60.2 years and there are 9 males and 5 female participants. For 1 patient we were unable to draw a baseline blood sample. Ninety three \% $(13 / 14)$ are diabetic, as evidenced by an approximate 2-fold elevation of the mean fasting blood glucose (reference values $3.6-6.0 \mathrm{mM}$ ) and an approximate 1.5 -fold increase in the mean values for HbA1c (reference value 4.0-6.0 \%). Sixty four \% (9/14) of the participants are undergoing dialysis for ESRD. Of the 13 baseline blood analysis, 2 participants have elevated total cholesterol levels (reference $<5.2 \mathrm{mM}$ ), 11 (85\%) participants have increased triglycerides, 3 (23\%) participants have increased total cholesterol/HDL-cholesterol levels (reference $<4.5 \mathrm{mM}$ ) and 2 (15\%) have elevated LDL-cholesterol/HDL-cholesterol levels (reference $<3.5$ 
$\mathrm{mM}$ ). Eight (62\%) participants have low HDL-cholesterol levels (reference $>1.0 \mathrm{mM})$. It should be noted that $85 \%(11 / 13)$ of the participants have elevated C-reactive protein levels (normal range $<5 \mathrm{mg} / \mathrm{L}$ ).

Table 4 shows some of the baseline limb characteristics of the participants. It can be seen that 29\% (4/14) patients are below knee amputees. All patients have at least one distal extremity ulcer. It should be noted that the mean value for $\mathrm{O}_{2}$ saturation at the site of the wound is around $40 \%$.

\section{TABLE 4}

\section{Baseline limb characteristics of patients enrolled into the Carbothera Study}

\begin{tabular}{ll}
\hline Amputees (n) & $\mathbf{3 6} \% \mathbf{( 5 )}$ \\
BKA $(\mathrm{n})$ & 4 \\
Toe $(\mathrm{n})$ & 1 \\
Number of wounds & 20 \\
$\% \mathrm{O}_{2}$ saturation at site of ulcer $(\mathrm{n})$ & $40.89 \pm 4.81(20)$ \\
Right Ankle Brachial Index & $0.87 \pm 0.09(8)$ \\
Left Ankle Brachial Index & $0.90 \pm 0.06(9)$ \\
Right side non compressibility $(\mathrm{n})$ & 2 \\
Left side non compressibility $(\mathrm{n})$ & 5
\end{tabular}

Values are means S.E. of indicated independent measurements. BKA, below knee amputation

Although 6 of the participants have peripheral arterial disease (left and/or right limb) as evidenced by an ankle brachial index (ABI) $<0.9$, specifically, $29 \%(4 / 14)$ of the participants have left side ABI mean value of 0.74 , and $36 \%(5 / 14)$ have a right side ABI mean value of 0.70 , which corresponds with mild to moderate obstruction of peripheral arteries in the limbs. It is pointed out that in 3 of the patients both right side and left side $A B I$ is $>1.0$, indicating no significant narrowing or blockage of peripheral blood flow. An $\mathrm{ABI}$ in either right and left side or both was not attainable in 5 participants due to non-compressibility of the peripheral arteries.

\section{DISCUSSION}

Many interventions have been proven with the objective of improving the healing process of diabetic and ischemic foot ulcers. Amongst others, the treatment includes: off-loading, wound debridement, control of gangrene or sepsis, antibiotic therapy and negative-pressure wound therapy. Nevertheless, these therapeutic measures can prosper only in the presence of adequate arterial perfusion (29). The Carbothera foot care study will generate data on the safety, tolerability, vascular efficacy and capacity to heal ulcerated foot lesions. It will provide data on the biochemical parameters that may be involved in the healing process. It will determine, for the first time, the effects of the Carbothera $\mathrm{CO}_{2}$ foot bath on primary events such as ulcer area (length $\mathrm{x}$ width), ulcer volume, (length $\mathrm{x}$ width $\mathrm{x}$ depth), the formation of new ulcers, ulceration rate and ankle-brachial index/Blood flow by Doppler (as shown in Table 2) as well as secondary endpoints such as the degree of rest pain, limb oxygenation $\left(\mathrm{O}_{2}\right.$ saturation), measurement of angiogenesis marker, inflammatory markers, fasting blood glucose, HbA1c, total cholesterol, HDL-cholesterol, LDL-cholesterol, triglycerides, use of bespoke footwear, ability to walk barefoot at home, incidence of amputation and use of antibiotics indicated for the ulcer treatment, in patients with ulcerated lesions (as shown in Table 2). The interest about $\mathrm{CO}_{2}$ as potential healing compound is based on its capacity as vasodilator of blood vessels and its capacity on improving the tissue perfusion in areas under perfused (10). The development by Mitsubishi Rayon Cleansui, Tokyo, Japan, of a gas permeable hollow fibre $\left(\mathrm{GPH}^{\mathrm{TM}}\right)$ allows the device Carbothera to generate, in a short time, large amounts of $\mathrm{CO}_{2}$. This capacity attracts the interest of having a small, practical device that enriches water with $\mathrm{CO}_{2}$ in order to treat foot ulcers at the bedside.

FUNDING: This study was supported by Mitsubishi Rayon Cleansui, Tokyo, Japan and St. Boniface Hospital Research Foundation, Winnipeg, Canada.

DISCLOSURES: Mitsubishi Rayon Cleansui is the manufacturer of the Carbothera device and provided their device for use in this study. Drs. Hasebe and Kumamoto are affiliated to Mitsubishi Rayon Cleansui. Drs. Tappia, Pierce and Ramjiawan are affiliated to the Albrechtsen Research Centre, St. Boniface Hospital. Infrastructural support for this study was also received from St. Boniface Hospital Research Foundation.

\section{REFERENCES}

1. Singh N, Armstrong DG, Lipsky BA. Preventing foot ulcers in patients with diabetes. JAMA 2005;293:217-28.

2. Boulton AJ, Vileikyte L, Ragnarson-Tenvall G, et al. The global burden of diabetic foot disease. Lancet 2005;366:1719-25.

3. Jeffcoate WJ, Harding KG. Diabetic foot ulcers. Lancet. 2003;361:1545-51.

4. Clayton W, Elasy TA. A review of the pathophysiology, classification, and treatment of foot ulcers in diabetic patients. Clin Diabetes 2009;27:52-58.

5. Lambert MA, Belch JJ. Medical management of critical limb ischaemia: where do we stand today?. J Intern Med 2013;274:295-307.

6. McGrath NM, Curran BA. Recent commencement of dialysis is a risk factor for lower-extremity amputation in a high risk diabetic population. Diabetes Care 2000;23:432-33.

7. Ndip A, Rutter MK, Vileikyte L, et al. Dialysis treatment is an independent risk factor for foot ulceration in patients with diabetes and stage 4 or 5 chronic kidney disease. Diabetes Care 2010;33:1811-16.

8. Game FL, Chipchase SY, Hubbard R, et al. Temporal association between the incidence of foot ulceration and the start of dialysis in diabetes mellitus. Nephrol Dial Transplant 2006;21:3207-10.

9. Beckert S, Sundermann K, Wolf S, et al. Haemodialysis is associated with changes in cutaneous microcirculation in diabetes mellitus. Diabet Med 2009;26:89-92.

10. Hartmann BR, Bassenge E, Hartmann M. Effects of serial percutaneous application of carbon dioxide in intermittent claudication: results of a controlled trial. Angiology 1997;48:957-63.

11. Hartmann BR, Bassenge E, Pittler M. Effect of carbon dioxide-enriched water and fresh water on the cutaneous microcirculation and oxygen tension in the skin of the foot. Angiology 1997;48:337-43.

12. Toriyama T, Kumada Y, Matsubara T, et al. Effect of artificial carbon dioxide foot bathing on critical limb ischemia (Fontaine IV) in peripheral arterial disease patients. Int Angiol 2002;21:367-73.

13. Nishimura N, Sugenoya J, Matsumoto T, et al. Effects of repeated carbon dioxide-rich water bathing on core temperature, Cutaneous blood flow and thermal sensation. Eur J Appl Physiol 2002;87:337-42.

14. Hayashi H, Yamada S, Kumada Y, et al. Short and long-term changes of the transcutaneous oxygen pressure $\left(\mathrm{tcPO}_{2}\right)$ during carbon dioxide foot bathing in patients with ischemic limbs. Jpn Coll Angiol 2006;46:411-16.

15. Makita S, Ohira A, Naganuma Y, et al. The effects on skin blood flow of immersing the schemic legs of patients with peripheral arterial disease into artificially carbonated water. Int J Angiol 2006;15:12-25.

16. Hayashi H, Yamada S, Kumada Y, et al. Immersing feet in carbon dioxideenriched water prevents expansion and formation of ischemic ulcers after surgical revascularization in diabetic patients with critical limb ischemia. Ann Vasc Dis 2008;1:111-17.

17. Alkhawaja S. Carbal therapy for treatment of diabetic foot $\left(\mathrm{CO}_{2}\right.$ water bath). Kufa Med J 2012;15:211-22.

18. Hunt DL. Diabetes: foot ulcers and amputations. BMJ Clin Evid 2011;2011:0602

19. Margolis DJ, Kantor J, Berlin JA. Healing of diabetic neuropathic foot ulcers receiving standard treatment. A meta-analysis. Diabetes Care 1999;22:69295.

20. Jeffcoate WJ, Chipchase SY, Ince P, et al. Assessing the outcome of the management of diabetic foot ulcers using ulcer-related and person-related measures. Diabetes Care 2006;29:1784-87.

21. Rogers LC, Bevilacqua NJ, Armstrong DG, et al. Digital planimetry results in more accurate wound measurements: a comparison to standard ruler measurements. J Diabetes Sci Technol 2010;4:799-802.

22. Xu D, Li J, Zou L, et al. Sensitivity and specificity of the ankle--brachial index to diagnose peripheral artery disease: a structured review. Vasc Med 2010;15:361-69.

23. Miura H, McCully K, Hong L, et al. Regional difference of muscle oxygen saturation and blood volume during exercise determined by near infrared imaging device. Jpn J Physiol 2001;51:599-606.

24. Shuler MS, Reisman WM, Whitesides TE Jr., et al. Near-infrared spectroscopy in lower extremity trauma. J Bone Joint Surg Am 2009;91:1360-68.

25. Wakimoto MM, Kasosaki M, Nagata $\mathrm{H}$, et al. The usefulness of near-infrared spectroscopy in the anesthetic management of endovascular aortic aneurysm repair. J Anesth 2012;26:932-35.

26. Fellahi JL, Butin G, Zamparini G, et al. Lower limb peripheral NIRS parameters during a vascular occlusion test: An experimental study in healthy volunteers. Ann Fr Anesth Reanim 2014;33:e9-e14.

27. Das S, Babick AP, Xu YJ, et al. TNF- $\alpha$-mediated signal transduction pathway is a major determinant of apoptosis in dilated cardiomyopathy. J Cell Mol Med 2010;14:1988-97.

28. Melzack R. The short-form McGill Pain Questionnaire. Pain 1987;30:191-197.

29. Kontopodis N, Tavlas E, Papadopoulos G, et al. Effectiveness of plateletrich plasma to enhance healing of diabetic foot ulcers in patients with concomitant peripheral arterial disease and critical limb ischemia. Int J Low Extrem Wounds 2015;15:45-51. 